\title{
Ethics check-up of public health immunization programs in Canada
}

\author{
Noni E MacDonald ${ }^{1 *}$, Shawn Harmon ${ }^{1,2}$, Janice E Graham ${ }^{1,3}$
}

\begin{abstract}
The World Health Organization (WHO) recognizes immunization as one of the most successful and effective public health interventions for saving lives. In developing a roadmap for prioritizing use of the coronavirus disease 2019 (COVID-19) vaccines in the context of limited supply, WHO highlighted the importance of a values (ethical principles) framework. Immunization does need to be subject to independent ethical scrutiny of vaccine research data, manufacturing practices, the legal and ethical assurance of informed consent, and also social justice issues with respect to program equity, including right to access. An ethics review of Australia's immunization program was reported in 2012. This CANVax (Canadian Vaccination Evidence Resource and Exchange Centre) Brief offers an ethics review of immunization in Canada using the criteria utilized for Australia.
\end{abstract}

Suggested citation: MacDonald NE, Harmon S, Graham JE. Ethics check-up of public health immunization programs in Canada. Can Commun Dis Rep 2021;47(4):237-41. https://doi.org/10.14745/ccdr.v47i04a09

Keywords: ethics, immunization, public health, health law, Canada

\section{Introduction}

The World Health Organization (WHO) recognizes immunization as one of the most successful and effective public health interventions for saving lives (1). The effects of immunization reach far beyond the health and well-being of individuals and communities by improving social determinants of health that affect work productivity, equity, institutional stability, economic development and innovation (see Figure 1). The coronavirus disease 2019 (COVID-19) pandemic has focused global attention on the importance of public health interventions and immunization to restore health and well-being of individuals and communities. In developing a roadmap for prioritizing use of COVID vaccines in the context of limited supply (2), WHO highlighted the importance of a values framework (3). This values framework draws from ethical principles focusing on human well-being, equal respect, global equity, national equity, reciprocity and legitimacy. These principles, while important for COVID-19 vaccines, apply to all immunization programs. Immunization needs to be subject to independent ethical scrutiny of vaccine research data and manufacturing practices, the legal and ethical assurance of informed consent, and social justice issues, including right to access.

In this Brief, we offer an "ethics check-up" of Canada's public health immunization programs and suggest some areas for further improvements.
This work is licensed under a Creative Commons Attribution 4.0 International License.
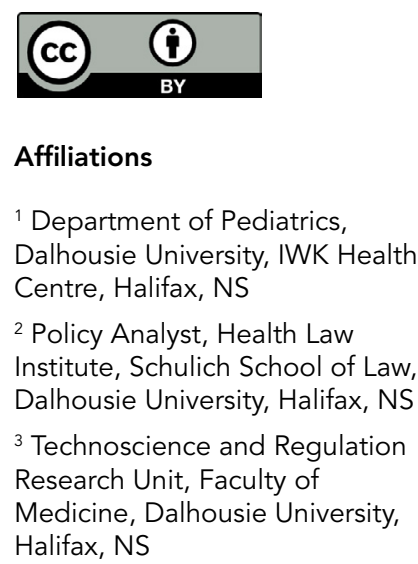

*Correspondence:

noni.macdonald@dal.ca

\section{Figure 1: Immunization and the Sustainable} Development Goals

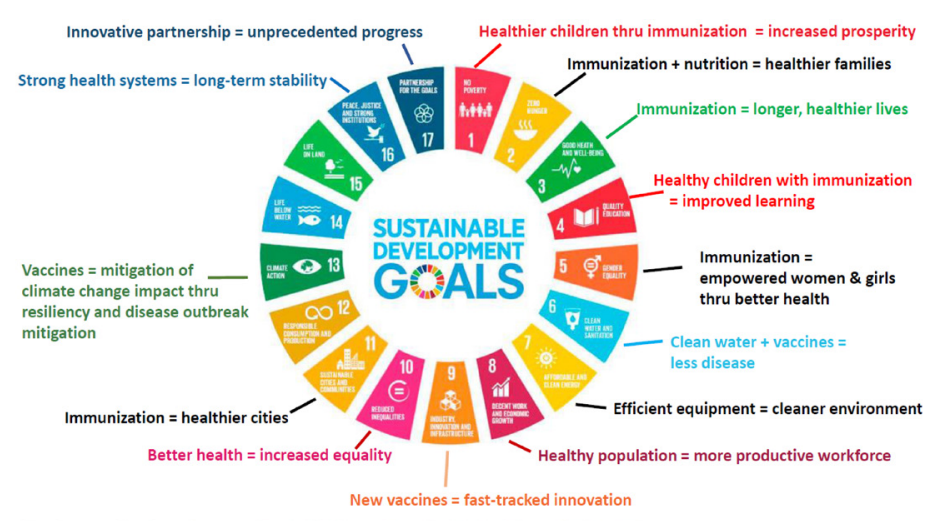

Adapted from GAVI - the Vaccine Alliance (https://www.gavi.org/about/ghd/sdg/)

\section{The check-up criteria used}

Immunization programs merit scrutiny for ethical practice not only by practitioners delivering the vaccines to patients but also to ensure ethical principles are followed over the whole program as the communities and populations targeted for immunization vary by age, disease burden, vulnerability and marginalization. 
The risks and benefits of immunization might vary in communities seen as vulnerable (e.g. infants, children, pregnant women, immunocompromised and older adults).

Attention to ethical issues has mainly focused on national risk/ benefit for policy recommendations and on trust through the lens of vaccine hesitancy/acceptance, while the ethical issues that arise at the front line (benefits, risks, effectiveness, equity and justice, autonomy, reciprocity and trust) have received less attention. Country-wide ethics assessment of immunization programs is a relatively nascent area.

Isaacs (4) developed seven ethical principles to inform assessments of public health immunization programs in Australia. These apply across the program from policy to frontline practices:

- Benefits

- Risks

- Effectiveness

- Equity and justice

- Autonomy

- Reciprocity

- Trust

This CANVax (Canadian Vaccination Evidence Resource and Exchange Centre) Brief applies Isaac's Australia ethics criteria (4) to Canada and draws attention to several concerns.

\section{Applying the criteria}

At the policy level, the National Advisory Committee on Immunization (NACl) and each of the provinces and territories make decisions on who is and who is not eligible for vaccines using an evidence-based framework. $\mathrm{NACl}$ is working to incorporate ethics, equity, feasibility and acceptability into their national recommendations (5). Assessment of the outcomes of the application of these ethical principles will only be known over time.

As noted, $\mathrm{NACl}$ provides evidence-based recommendations that take into account population burden and risks of disease, including the safety and expected benefits of the vaccine in different populations in Canada. The provinces and territories then make their public health policy decisions about including and paying for vaccine(s) in their immunization programs, for whom it will be made available, how the program will be delivered and whether catch-up programs will be introduced. (This does not include COVID-19 vaccines, for which the federal government is paying.)

Not all vaccines recommended by $\mathrm{NACl}$ are equally accessible across the country. Provincial differences in implementation can foster provider and public confusion, raising national equity and social justice concerns to do with access. For example, introduction of conjugated pneumococcal vaccine and varicella vaccine varied across the country, despite that both vaccine-preventable diseases (VPDs) are prominent everywhere (6). The disparity in timing of vaccine introduction continues, with Alberta introducing a school-based human papillomavirus (HPV) vaccine program for girls in 2008 and Nunavut only doing this in 2010 . Not all provinces and territories offer HPV vaccine catch-up programs for boys, and Nova Scotia's approval of routine rotavirus vaccine in their infant program only came in 2019, years after other provinces (7). Thus, access to $\mathrm{NACl}$-recommended vaccines varies depending on where one lives in Canada, underscoring equity and social justice issues.

\section{Risk, benefits and effectiveness}

Both the benefits of immunization, i.e. prevalence of VPD, and risks of immunization, i.e. adverse events following immunization (AEFI) are tracked in Canada through surveillance systems. Canada has both passive and active AEFI (8) and VPD reporting, with VPD reporting from laboratories now more automated than in the past. VPD and AEFI summary findings are regularly reviewed and discussed within the provinces or territories where they are reported as well as nationally at $\mathrm{NACl}$ and the Public Health Agency of Canada (PHAC). There are many examples where the introduction of a new vaccine across Canada has led to a marked decrease in the VPD, e.g. invasive Haemophilus influenzae type b (9) and pneumococcal-related diseases (10), providing data on vaccine effectiveness in Canadian populations. IMPACT (Immunization Monitoring Program ACTive), Canada's paediatric hospital-based national active surveillance network, which looks for vaccine failures, serious AEFI (i.e. hospitalization) and selected VPD and infectious diseases, has provided much valuable data on the effects of pediatric vaccines over the past two decades. Similar data for the effects of adult vaccines are not routinely collected. The Serious Outcomes Surveillance (SOS) Network collects data on adults admitted to hospital with influenza or pneumonia, but it does not track serious AEFI (11). Neither of these systems collect immunization uptake data for the specific region served by the reporting site, meaning that the effectiveness of the different vaccines locally can only be estimated. The CANImmunize app tailored for COVID-19 vaccines addresses some of these deficits but is not used fully across the country (12).

Vaccine failures are less well tracked. VPD-microbe reporting by microbiology laboratories is increasingly active across the country as they become automated. However, the detected VPD cases may lack information on whether this is a vaccine failure. The need to detect COVID-19 vaccine failures-due to the failure of the vaccine itself and due to program errors such as mishandling of vaccine storage and missed populations/subgroups-in order to establish effectiveness in different age groups and settings has pushed all the provinces and territories to collect immunization data for these vaccines so that vaccine-receipt failures as well as immunization failures can be more readily detected. However, not all regions have quality immunization registries for all vaccines. Overall, the principles of risk and effectiveness could be more broadly and better addressed across all age groups. 


\section{Autonomy and informed consent}

With respect to autonomy, school-based immunization programs raise unresolved consent and assent issues. While such programs are routinely offered across Canada, the age varies, from Grade 4 (approximately 10-11 years old) to Grade 9 (approximately 15-16 years old). Typically, parents sign a consent form for the vaccines to be administered, but if there is no consent and the student wants to be immunized, or if the consent and the student's stated decision are at odds, public health practitioners may be unclear about how to proceed and jurisdictional practices differ (13). In the case of positive parental consent with a student refusal, the vaccination is unlikely to proceed. But for the student who expresses a desire to be vaccinated in the face of parental refusal, there is more ambiguity. This uncertainty is confounded by variation across Canada at which age "capacity" is assumed, and the differences in approaches to "mature minors" (14). In Ontario, for example, there is no specific age at which a minor's capacity is assumed; children are assessed on their capacity to give consent, and this is informed less by their age and more by their understanding, which the healthcare provider must assess (15). Furthermore, some healthcare workers will not disregard the parental veto on immunization even if the minor appears to have capacity. A national standard of practice has not yet been established, which may impinge on adolescents' rights and undermine ethical consent standards.

Another uncertainty associated with informed consent involves vaccine information meeting user needs. In Canada, each province and territory develops their own patient/parent information packages. While these may draw on $\mathrm{NACl}$ information and advice, they often differ, leaving uncertainty as to whether the same vaccine is being described (16). For those seeking to make an informed decision, this can be confusing, if not off-putting, especially when proponents of no vaccination ("antivaxxers") state that the provided information is inadequate. Improving collaboration on developing materials that provide information about vaccines could make this area less confusing and contentious for those seeking information and may improve coverage in populations where new data confirm safety and effectiveness.

\section{Reciprocity}

Canada has a mixed picture of reciprocity for public health immunization programs. Hospital care costs associated with rare serious $\mathrm{AEFI}$ are covered by the publicly funded healthcare system. Any long-term disabilities caused by an AEFI may be supported, in part, by disability benefits offered through ministries of community and social services in the provinces and territories. However, these benefits usually do not cover lifelong loss of income due to inability to work. But vaccine causality is not always easy to assess. For example, causality assessment of narcolepsy incidents in Finland and Sweden after administration of the $\mathrm{H} 1 \mathrm{~N} 1$ vaccine is confounded by the known increased risk of narcolepsy with H1N1 infection, with the virus already circulating in the community when the immunization programs began (17). Disentangling the effect of natural infection concurrent with immunization is not simple. The onset of narcolepsy in childhood has a profound effect on education attainment, capacity for work and many social, family and personal outcomes. There are strong ethical arguments that such serious AEFls, when due to immunization, should be compensated, especially if vaccines are mandatory (18). In Canada, only Québec has a vaccine injury compensation program, in place since 1987 (19). In December 2020, Canada announced that PHAC is implementing a no-fault vaccine injury support program for all vaccines approved by Health Canada (20), but details are pending as of April 2021. This is a complex undertaking: there are seven characteristics essential to ensure fair, transparent and efficient operation of a vaccine injury compensation program (21).

\section{Trust, social justice and equity}

Issues of trust may arise for public immunization programs in a number of different areas. A cluster of AEFls may undermine trust in the program and in the vaccine if their causality is not addressed quickly and satisfactorily. This occurred in Japan and in Denmark with human papilloma vaccine $(22,23)$. It is unclear if all the provinces and territories or PHAC have serious AEFI rapid science review procedures and communication templates in place, or if the coordination between the government levels across the country is good. Of note, the last reported biannual update on the Canadian Adverse Events Following Immunization Surveillance System (CAEFISS) is for the period January to June 2019 (24). While the COVID-19 pandemic may have delayed further updates, it is disturbing that no new summary report has become available for AEFls in the past 18 months.

Providing mixed messages on AEFls, whether vaccine related or a coincidence, can rapidly undermine public trust (25). The media attention when Norway noted deaths in frail elderly people following COVID-19 vaccination illustrates how trust may be easily undermined (26).

Trust concerns also arise when different vaccines are available for the same disease but some are funded and other ones are not (e.g. different influenza vaccines targeting seniors) (27). This also raises ethical issues about cost-effectiveness assessment, social justice and the valuation of equity. Canada's contribution to COVAX, the global sharing of COVID-19 vaccines, followed by Canada's plan to receive vaccines from COVAX, unlike many other high-income countries, has raised concerns about Canada's commitment to global vaccine equity (28). Again, communication is key. We need discussions about public values and priorities that include voices from diverse communities. Moreover, evidence that these voices are valued and taken into consideration must be shown, or trust will be undermined. 


\section{Ethical considerations for healthcare practitioners who provide vaccination}

Public health nurses and physicians and their office staff represent the front line interacting with patients and caregivers in vaccination situations. The Canadian Medical Association Code of Ethics and Professionalism (29) and the Canadian Nurses Association Code of Ethics for registered nurses (30) articulate the ethical and professional commitments and responsibilities of the medical and nursing professions. The codes provide standards of ethical practice to guide physicians and nurses in fulfilling their obligation to provide the highest standard of care and to foster patient and public trust in the medical professions.

The Codes are not exhaustive; they are intended to provide standards of ethical practice that can be interpreted and applied in particular situations. How these could be applied to vaccination situations, especially for COVID-19 vaccines in these times of vaccine constraints, has not been well articulated.

\section{Conclusion}

Overall, this high-level ethics check-up of Canadian public health immunization programs suggests that Canada is generally on the right track, although there is room for improvement. Canada has "pass marks" in relation to principles such as benefits, risk and effectiveness. There are gaps and uncertainties to do with other principles, however (i.e. equity, justice, autonomy, reciprocity, trust). These speak to a need for greater attention to matters affecting other principles, including solidarity and transparency. The COVID-19 vaccine constraint context has further exacerbated some of these concerns.

Universal solutions are not easy given Canada's size and health system structure; regional differences in prevalence and burden of VPD result in different decisions. Provincial and territorial priorities in health care also result in variation in implementation. Having acknowledged this, readily justifiable steps that can improve collective coherence across immunization settings in Canada can be put into place in the short term; fragmentation and duplication are problematic. For example, providing a simple ethics self-assessment questionnaire for provinces and territories might identify both common and region-specific challenges and stimulate development of effective shared tools and responses. In addition, jointly developed best practices for issues such as consent and assent in school-based immunization programs would have country-wide benefits. Finally, a comprehensive review of serious AEFIs and the development of a template for AEFI crisis communication are undeniably valuable and within existing capacities. COVID-19 vaccine mass rollouts have added the pressure on this. Leadership is needed to tackle these challenges so that the results of the next ethics check-up-which might consider solidarity and transparency—might be improved.

\section{Authors' statement}

NEM - Conceptualization, writing original draft

$\mathrm{SH}-$ Conceptualization, revision of drafts, agreement with final draft

JEG - Conceptualization, revision of drafts, agreement with final draft

\section{Competing interests}

None.

\section{Funding}

No external funding.

\section{References}

1. Strategic Advisory Group of Experts on Immunization. Immunization today and in the next decade: 2018 Assessment report of the Global Vaccine Action Plan. Geneva: World Health Organization; 2018. https://www.who.int/immunization/global_ vaccine_action_plan/SAGE_GVAP_Assessment_Report_2018_ EN.pdf?ua $=1$

2. SAGE Working Group on COVID-19 Vaccines. WHO sage roadmap for prioritizing uses of COVID-19 vaccines in the context of limited supply: an approach to inform planning and subsequent recommendations based upon epidemiologic setting and vaccine supply scenarios. Version 1.1. Geneva: World Health Organization; 2020. https://www.who.int/docs/ default-source/immunization/sage/covid/sage-prioritizatio n-roadmap-covid19-vaccines.pdf?Status $=$ Temp\&sfvrsn $=$ bf227443_2

3. SAGE Working Group on COVID-19 Vaccines. WHO SAGE values framework for the allocation and prioritization of COVID-19 vaccination. Geneva: World Health Organization; 2020. https://apps.who.int/iris/bitstream/ handle/10665/334299/WHO-2019-nCoV-SAGE Framework-Allocation_and_prioritization-2020.1-eng. pdf?ua $=1$

4. Isaacs $D$. An ethical framework for public health immunisation programs. N S W Public Health Bull 2012;23(5-6):111-5. DOI PubMed

5. National Advisory Committee on Immunization. National Advisory Committee on Immunization (NACl): Workplan for 2019-2020. Ottawa (ON): Government of Canada; (modified 2020-04-22; accessed 2021-03-31). https://www.canada.ca/ en/public-health/services/immunization/national-advisor y-committee-on-immunization-naci/workplan.html

6. Halperin SA, Pianosi K. Immunization in Canada: a 6-year update. J Can Chiropr Assoc 2010;54(2):85-91. PubMed

7. Provincial and territorial routine and catch-up vaccination schedule for infants and children in Canada. Ottawa (ON): Government of Canada; (modified 2020-12-11; accessed 2021-03-21). https://www.canada.ca/en/public-health/ services/provincial-territorial-immunization-information/ provincial-territorial-routine-vaccination-programs-infantschildren.html 
8. MacDonald NE, Law BJ. Canada's eight-component vaccine safety system: A primer for health care workers. Paediatr Child Health 2017;22(4):e13-6. DOI PubMed

9. Scheifele DW. Recent trends in pediatric Haemophilus influenzae type B infections in Canada. Immunization Monitoring Program, Active (IMPACT) of the Canadian Paediatric Society and the Laboratory Centre for Disease Control. CMAJ 1996;154(7):1041-7. PubMed

10. Kellner J; Canadian Paediatric Society, Infectious Diseases and Immunization Committee. Update on the success of the pneumococcal conjugate vaccine. Paediatr Child Health 2011;16(4):233-40. DOI PubMed

11. Canadian Immunization Research Network(CIRN). Serious Outcomes Surveillance (SOS) Network: what is SOS. Halifax (NS): Canadian Immunization Research Network; (accessed 2021-03-21). http://cirnetwork.ca/network/serious-outcomes/

12. CANImmunize. Digitally transforming immunization practice for government, employers, health care and Canadians. Ottawa (ON): CANimmunize; (accessed 2021-04-15).

https://www.canimmunize.ca/en/home

13. Glauser W. Teens, vaccines and the age of consent. CMAJ 2019;191(12):E348-9. DOI PubMed

14. Coughlin KW; Canadian Paediatric Society Committee on Bioethics. Medical decision-making in paediatrics: infancy to adolescence. Paediatr Child Health 2018;23(2):138-46. DOI PubMed

15. Schwartz Y, Williams TS, Roberts SD, Hellmann J, Zlotnik Shaul R. Adolescent decision-making in Canadian medical contexts: integrating neuroscience and consent frameworks. Paediatr Child Health 2018;23(6):374-6. DOI PubMed

16. Steenbeek A, Macdonald N, Downie J, Appleton M, Baylis F. Ill-informed consent? A content analysis of physical risk disclosure in school-based HPV vaccine programs. Public Health Nurs 2012;29(1):71-9. DOl PubMed

17. Sarkanen TO, Alakuijala AP, Dauvilliers YA, Partinen MM. Incidence of narcolepsy after H1N1 influenza and vaccinations: systematic review and meta-analysis. Sleep Med Rev 2018;38:177-86. DOI PubMed

18. Attwell K, Drislane S, Leask J. Mandatory vaccination and no fault vaccine injury compensation schemes: an identification of country-level policies. Vaccine 2019;37(21):2843-8.

DOI PubMed

19. Government of Quebec. Vaccine Injury Compensation Program. Québec (QC): Government of Quebec; (updated 2021-03-17; accessed 2021-03-31). https://www.quebec.ca/en/health/advice-and-prevention/ vaccination/vaccine-injury-compensation-program

20. Public Health Agency of Canada. Government of Canada announces pan-Canadian vaccine injury support program. News release. December 10, 2020 - Ottawa (ON): PHAC; (accessed 2021-03-31). https://www.canada.ca/en/public-health/ news/2020/12/government-of-canada-announces-pan-canadi an-vaccine-injury-support-program.html
21. Harmon $\mathrm{SH}$. Characteristics of a fair vaccine injury compensation program for Canada. RSC COVID-19 Series. Publication \#87. Ottawa (ON): Royal Society of Canada; 2021 (accessed 2021-04-15). https://rsc-src.ca/en/voices/characteristics-fai r-vaccine-injury-compensation-program-for-canada

22. Ikeda SI, Hineno A, Ozawa K, Kinoshita T. Suspected adverse effects after human papillomavirus vaccination: a temporal relationship. Immunol Res 2018;66(6):723-5. DOI PubMed

23. Baumann A, Andersen B, Østergaard L, Larsen MB. Sense \& sensibility: decision-making and sources of information in mothers who decline HPV vaccination of their adolescent daughters. Vaccine X 2019;2:100020. DOI PubMed

24. Canadian Adverse Events Following Immunization Surveillance System (CAEFISS). Ottawa (ON): Government of Canada; (modified 2019-12-11; accessed 2021-03-31).

https://www.canada.ca/en/public-health/services/ immunization/canadian-adverse-events-follow ing-immunization-surveillance-system-caefiss.html

25. World Health Organization. Vaccination and trust: how concerns arise and the role of communication in mitigating crises. Copenhagen (DK): WHO; 2017. https://www.euro.who.int/ data/assets/pdf_file/0004/329647/Vaccines-and-trust.PDF

26. Kottasova I, Dean S, Sealy A. Norway reviewing deaths of frail and elderly patients vaccinated against COVID-19. Toronto (ON): CTV News; 2021-01-19 (updated 2021-01-19; accessed 2021-03-31). https://www.ctvnews.ca/health/coronavirus/ norway-reviewing-deaths-of-frail-and-elderly-patients-vaccin ated-against-covid-19-1.5272547

27. Vaudry W, Zhao L, Stirling R; National Advisory Committee on Immunization (NACl). Summary of the $\mathrm{NACl}$ Seasonal Influenza Vaccine Statement for 2018-2019. Can Commun Dis Rep 2018 Jun;44(6):123-8. DOI PubMed

28. Major D, Cullen C. Canada shouldn't take vaccine doses from COVAX partnership, says Canada's former UN envoy. CBC News; (updated 2021-02-26; accessed 2021-04-18). https://www.cbc.ca/news/politics/canada-stephe n-lewis-covax-1.5930344\

29. Canadian Medical Association. CMA code of ethics and professionalism. Ottawa (ON): CMA; 2018. https://www.cma. $\mathrm{ca} / \mathrm{cma}$-code-ethics-and-professionalism

30. Canadian Nurses Association. Code of ethics for registered nurses, 2017 edition. Ottawa (ON): CNA; 2017. https://www.cna-aiic.ca/ /media/cna/page-content/pdf-en/ code-of-ethics-2017-edition-secure-interactive 